\title{
Virtual/Real Transfer in a Large-Scale Environment: Impact of Active Navigation as a Function of the Viewpoint Displacement Effect and Recall Tasks
}

\author{
Grégory Wallet, ${ }^{1}$ Hélène Sauzéon, ${ }^{2,3}$ Florian Larrue, ${ }^{2}$ and Bernard N'Kaoua ${ }^{2,3}$ \\ ${ }^{1}$ Aix-Marseille Université, CNRS, ISM UMR 7287, 13288 Marseille Cedex 09, France \\ ${ }^{2}$ Université Victor Segalen Bordeaux II, Laboratoire Handicap et Système Nerveux EA 4136, 33076 Bordeaux Cedex, France \\ ${ }^{3}$ INRIA, PHOENIX Team, Bordeaux Sud-Ouest, 400 Avenue de la Vieille Tour, 33400 Talence, France \\ Correspondence should be addressed to Grégory Wallet; gregwallet@free.fr
}

Received 14 May 2013; Revised 13 August 2013; Accepted 27 August 2013

Academic Editor: Ian Oakley

Copyright (C) 2013 Grégory Wallet et al. This is an open access article distributed under the Creative Commons Attribution License, which permits unrestricted use, distribution, and reproduction in any medium, provided the original work is properly cited.

\begin{abstract}
The purpose of this study was to examine the effect of navigation mode (passive versus active) on the virtual/real transfer of spatial learning, according to viewpoint displacement (ground: $1 \mathrm{~m} 75$ versus aerial: $4 \mathrm{~m}$ ) and as a function of the recall tasks used. We hypothesize that active navigation during learning can enhance performances when route strategy is favored by egocentric match between learning (ground-level viewpoint) and recall (egocentric frame-based tasks). Sixty-four subjects (32 men and 32 women) participated in the experiment. Spatial learning consisted of route learning in a virtual district (four conditions: passive/ground, passive/aerial, active/ground, or active/aerial), evaluated by three tasks: wayfinding, sketch-mapping, and picture-sorting. In the wayfinding task, subjects who were assigned the ground-level viewpoint in the virtual environment (VE) performed better than those with the aerial-level viewpoint, especially in combination with active navigation. In the sketch-mapping task, aerial-level learning in the VE resulted in better performance than the ground-level condition, while active navigation was only beneficial in the ground-level condition. The best performance in the picture-sorting task was obtained with the ground-level viewpoint, especially with active navigation. This study confirmed the expected results that the benefit of active navigation was linked with egocentric frame-based situations.
\end{abstract}

\section{Introduction}

Spatial cognition refers to the cognitive processes associated with the development of a comprehensive understanding of a spatial environment and the utilization of that knowledge for various purposes. Large-scale spatial cognition provides procedural knowledge and/or configurational knowledge about the environment which results from the acquisition of spatial knowledge levels [1, 2]: landmarks, route, and survey knowledge of the environment. The third level would allow the development of a cognitive map that contains configurational information. This L-R-S model (landmarks, route, and survey) has been subsequently described in a "seriparallel" form, in which the initiation of the construction of configurational knowledge may start independently from the completion of subordinate levels $[3,4]$.
In everyday life navigation, individuals use two types of strategies: route strategy and survey strategy. Each strategy is based on the reference frame of the internal representations developed during navigation, that is, egocentric representation (body-centered) in route strategy and allocentric representation (world-centered) in survey strategy [5]. The frame of reference of the internal representations (i.e., egocentric versus allocentric) often depends on the learned viewpoint $[6,7]$. In other words, different strategies will be involved depending on the different types of learning sources used $[8,9]$. For example, direct navigation involves egocentric representations and facilitates understanding of the route, while studying a map involves allocentric representations and promotes the acquisition of survey knowledge [8].

Research into spatial cognition has benefited from the introduction of Virtual Reality (VR) technology [10]. 
However, it is important to assess similarities and differences between knowledge obtained in real life and that obtained in virtual environments (i.e., to verify that the best transfer of knowledge from the VE to the real situation is obtained) [11]. Transfer studies make a distinction between transfer of skill (from one sensory modality to another) and transfer of spatial knowledge (knowledge conservation from learning to test situation) [11]. Several studies have demonstrated an effective transfer of skill and/or spatial knowledge from virtual to real environments (virtual/real transfer), indicating that the spatial knowledge acquired in virtual environments is quite similar to that acquired in real environments (e.g., [11-20]). With respect to activities of daily living (ADL), the aim of the "transfer" studies is to measure the benefits of the exposure to the VE on the future navigation activities in real life. Such a task is classically called "wayfinding." It consists of verifying that a subject can replicate in a real situation a route learned in VE. Moreover, various additional factors can optimize the quality of this type of transfer (e.g., [18-20]).

Among factors that have mobilized the researcher interest is the user navigation mode relative to the spatial cognition field. Evidence from experiments in a real environment have indicated that spatial learning performance improved when subjects navigated actively (i.e., active condition) compared to passive navigation (i.e., the subject was only an observer). Active navigation may therefore reinforce perception-action coupling (e.g., $[21,22])$. In VR studies and according to the taxonomy presented by Wilson et al. [23] four navigation modes are distinguished: (i) physically passive navigation (the subject observes the VE without moving), (ii) physically active navigation (the subject moves using a motor interactor), (iii) psychologically passive navigation (the subject follows directions), and (iiii) psychologically active navigation (the subject freely explores the VE). In the present study, we focus on the physical navigation mode. Some earlier studies in VR have demonstrated that active physical navigation was more effective for spatial knowledge acquisition (e.g., [24-26]), whereas others revealed an inferior effect, or no significant difference at all (e.g., [23, 27-30]). Also, other studies have obtained mixed results, depending on the type of recall task involved (e.g., [13, 18-20, 31, 32]). Thus, the beneficial effect of active navigation in VE studies has not yet been clearly established. This can be due to the fact that active navigation is examined by these authors in different experimental designs, mode of navigation, and test administered (see [33] for review).

Few studies have examined the impact of active navigation on the virtual/real transfer of skill (in using wayfinding task) (e.g., [18-20, 32]). These studies suggest a beneficial effect of the active navigation. For example, Wallet et al. [20] studied the effect of active learning in VE on performances in wayfinding task. This task is considered as principally based on route strategy when it comes to path following after route learning [2]. The subjects had to learn a route in a virtual environment in passive or active mode (with or without joystick) and then replicate this route in the real environment. The results indicate better performances for "active" participants. These results can be explained, as for real environment results, by a consolidation of the perception-action coupling (e.g., $[20,24]$ ) (leading to the route strategy in pedestrian navigation). The two strategies are probably used for navigation of daily living as individuals can "switch" between egocentric and allocentric representations during navigation [34]. However, these results [20] suggest that a strategy is privileged according to the situation. Consequently, we presume that the activity during navigation is beneficial particularly in egocentric frame-based situation. To test this hypothesis, we propose to disrupt the frame of reference in manipulating the viewpoint (during active learning in VR).

In VR-based studies, the viewpoint is easily manipulated according to the locomotion metaphor implemented to display the VE. Ground- or aerial-level metaphors are simply display parameters of VE. Darken and Peterson [35] claimed that the viewpoint displacement metaphor was an important variable in VR systems, due to its mediating effects on spatial representation and understanding of the VE explored. Learning in ground-level mode involves egocentric representations and facilitates understanding of the route, whereas the aerial-level mode can involve allocentric representations and promotes the acquisition of survey knowledge [36]. For example, Witmer et al. [36] proposed several navigation aids to evaluate the acquisition of configuration knowledge. Among these aids, the authors proposed adding an aeriallevel mode (such as map in this case) to ground-level navigation in VE. The results obtained indicate that the addition of a map during navigation improves performance in tasks on the environment configuration. In our experiment, for aerial-level we apply a 4 meter elevation to the viewpoint. It is difficult to envisage that a $4 \mathrm{~m}$ high viewpoint would lead to a "total" survey representation; however, we expect that this unusually elevated perspective will be sufficient to disturb the acquisition of egocentric representation during active navigation.

In this context, the main purpose of this study was to investigate the impact of navigation mode (physically passive versus active) on the virtual/real transfer of spatial learning according to viewpoint displacement (ground: $1 \mathrm{~m} 75$ versus aerial: $4 \mathrm{~m}$ ). Our main task involves wayfinding. It consists in verifying that a subject can replicate in a real situation a route learned in VE. This task is probably the closest one to the everyday life spatial activities and allows us to examine the virtual/real transfer of skills. We added two laboratory tasks (sketch-mapping task and picture-sorting task) to study the transfer of spatial knowledge and to study the impact of the type of spatial recall task administered. Concerning this last point, several authors have mentioned that the outcome of active-passive comparisons may depend on the type of spatial recall task and the measure employed (e.g., [13, 33]). We hypothesized that an active mode of route learning may enhance perception-action coupling (leading to the route strategy in pedestrian navigation) in an egocentric frame-based situation. In other words, we expect that active navigation may be beneficial when route strategy is favored by a matched viewpoint between learning (ground-level viewpoint) and recall (egocentric frame-based tasks). Thus, we expect the best performances in wayfinding task to be obtained in active/ground-level navigation. 


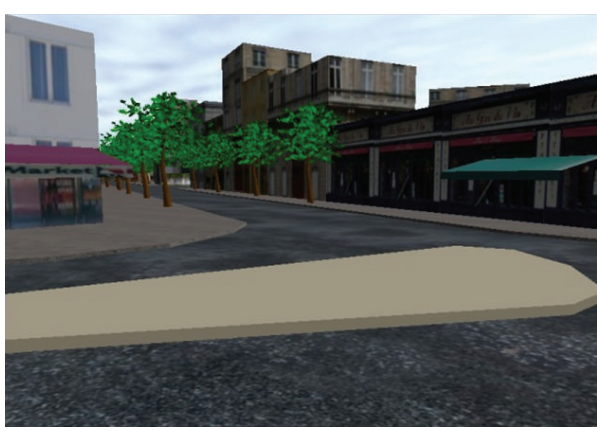

(a)

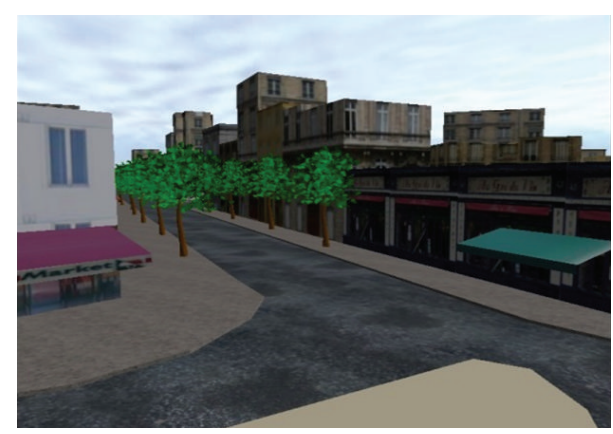

(b)

FIGURE 1: Pictures of the same spot in virtual environment, from the ground-level condition (a) and from the aerial-level condition (b).

Concerning the laboratory tasks, we hypothesized that the active/ground-level navigation would facilitate the performance in the picture-sorting task (egocentric frame-based) and passive/aerial-level navigation in the sketch-mapping task (allocentric frame-based). For these hypotheses, the "frame" does not refer to the internal representation but rather just to the frame of reference proposed by tasks.

\section{Method}

2.1. Participants. The participants were 64 student volunteers from Victor Segalen Bordeaux 2 University (32 men and 32 women, with an average age of 20). All subjects were unfamiliar with the area used in experimentation. All of the subjects were native French speakers and right-handed. Thirty-two subjects participated in the ground-level condition and 32 in the aerial-level condition (corresponding to the type of viewpoint displacement). In each condition, two groups of 16 subjects were formed according to the type of learning (i.e., passive virtual versus active virtual). The four groups were matched by age $(P>.200)$ and number of years of education $(P>.800)$. To match the groups in terms of spatial skills, the subjects performed paper-pencil tests before the experimental tasks. Spatial skills were assessed using the following: the Survey Aptitude Test (only parts GZ-5 and GZ-6; [37]) and the Mental Rotation Test (MRT, [38]). There were no significant differences between the groups in terms of performance on any of the spatial tests $(P>.200 ; P>.900$; $P>.900$, resp.). In addition, there were no gender differences across the groups for the two parts of the Survey Aptitude Test $(P>.900 ; P>.400$, resp. $)$ or the MRT $(P>.600)$.

2.2. Material. Two environments were used in our experiment: virtual and real. The real environment was an area near the Bordeaux hospital, and the virtual environment (VE) was a 3D replica of that environment created in 2006 by engineering students from Bordeaux 2 University, using Virtools software. Significant landmarks (e.g., signposts, signs, urban furniture, etc.) as well as an auditory environment (i.e., urban sounds) were included in the VE. The characteristics of the route were as follows: 1,457 meters, 14 streets, 18 intersections, and 18 direction changes. To manipulate the navigation mode in the VE in the passive condition, participants only visualized the route without any interaction, whereas in the active condition, the participants used a joystick to interact with the VE. In this present experiment, with the future aim of developing a retraining program for patients with orientation disorders, we selected a joystick, because it is an interactor suitable for this population [39]. This allows using it with patients who present a lower limb motor disability at the time of cognitive rehabilitation.

To manipulate the viewpoint mode in the $\mathrm{VE}$ in the ground-level condition, participants visualized the route at human height (i.e., 1.75 meters above the ground), whereas in the aerial-level condition, the participants visualized the route according to an elevated viewpoint (i.e., 4 meters above the ground). In these two viewpoints (see Figure 1) conditions, the eye orientation was parallel with the ground plane.

The apparatus used in the virtual reality room was a Dell personal computer ( $3 \mathrm{GHz}, 5 \mathrm{~GB}$ RAM) with an nVidia Quadro FX 4400 graphics card, F1+ projector, $2 \times 1.88$ meter flat screen, and Logitech force 3D pro joystick for the active navigation mode. The participants were seated two meters from the display screen.

2.3. Procedure. The experimental procedure consisted of the three following steps for each participant: (1) a training phase (10 min.): the subject freely explored a virtual environment similar to the VE used in the experiment (created by the same process, but representing a different area of Bordeaux). The purpose of this phase was to allow the subjects to become comfortable with using the VE technology. (2) A route learning phase: participants were randomly allocated to groups. They visualized either the ground-level viewpoint or the aerial-level viewpoint. In each condition, the participants were divided into two groups according to the display mode: a passive virtual environment (with a recorded route and directions given by the researcher) or an active virtual environment with a joystick (the route was still pre-established by the researcher who gave direction instructions). (3) A test phase: three spatial knowledge recall tasks presented in a counterbalanced order among the participants.

(i) The wayfinding task was a reproduction of the route in the real environment. Participants were required 


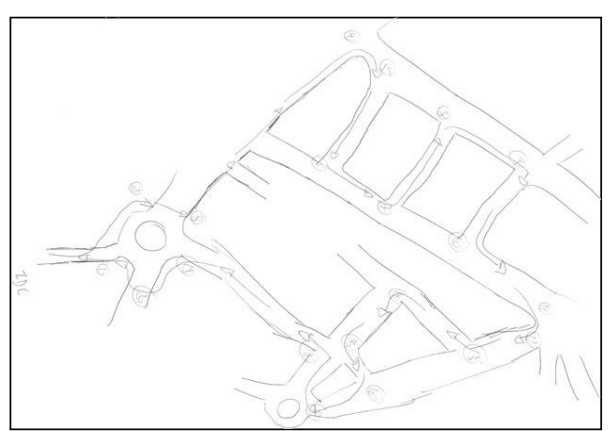

(a)

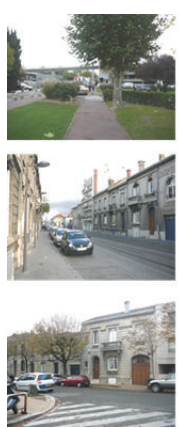

21.

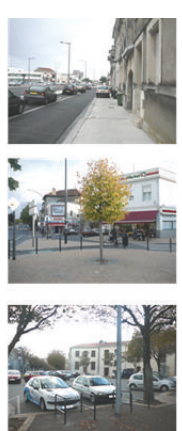

(b)

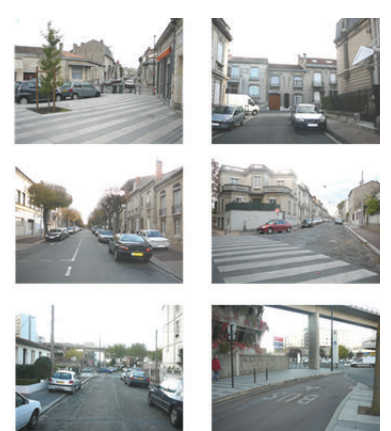

FIGURE 2: Illustration of the type of result obtained on the sketch-mapping task (a) and of the material used on the picture-sorting task (b) during the recall phases.

to replicate the learned route in real life. Direction errors were noted. In case of error, participants were stopped and asked to change direction. In some cases, participants made several directional errors at the same intersection. In this task, all errors in direction changes were counted.

(ii) The sketch-drawing task consisted of a free-hand reproduction of the visualized route in the form of a sketch (see Figure 2(a)). Participants were required to draw a simple outline sketch (connected segments), and the goal was to indicate and number the direction changes. In this task, all errors and omissions in direction changes on the sketches were counted.

(iii) The picture-sorting task consisted of sorting a series of pictures (in real world) taken along the route into encountered order (see Figure 2(b)). The experimenter counted the number of classification errors (i.e., incorrectly positioned pictures).

\section{Results}

A two-way MANOVA (Navigation (passive virtual versus active virtual) $\times$ Viewpoint (ground versus aerial)) was applied to the three dependent measures from the wayfinding, sketch-mapping, and picture-sorting tasks. This multivariate analysis revealed significant effects for each main factor and their interaction as follows: Navigation, $\lambda^{2}=.816$, $F(3,58)=4.37, P<.01$; Viewpoint, $\lambda^{2}=.496, F(3,58)=$ 19.63., $P<.0001$; and their interaction, $\lambda^{2}=.355, F(3,58)=$ $35.13, P<.0001$.

An examination of the univariate effects produced the following results. All means and standard deviations are reported in Figure 3 (the score for each task was compared with the best potential score to obtain percentages).

3.1. Direction Errors in the Wayfinding Task in Reality. The ANOVA analysis revealed a significant effect for the following:

(i) navigation factor $[F(1,60)=5.831 ; P<.05]$ : subjects performed less well in the active than the passive navigation condition; (ii) viewpoint factor $[F(1,60)=18.832 ; P<.0001]$ : subjects performed better in the ground-level than the aerial-level viewpoint condition;

(iii) "navigation $\times$ viewpoint" interaction $[F(1,60)=$ 25.396; $P<.0001]$ : subjects performed better in active than passive navigation in the ground-level viewpoint (i.e., positive effect of active condition); thus the reverse was observed with the aerial-level viewpoint (i.e., a negative effect of the active condition).

3.2. Errors and Omissions in the Sketch-Mapping Task. The ANOVA analysis revealed a significant effect for the following:

(i) navigation factor $[F(1,60)=4.188 ; P<.05]$ : subjects performed better in the active than the passive navigation condition;

(ii) viewpoint factor $[F(1,60)=6.256 ; P<.05]$ : subjects performed better in the aerial-level than the groundlevel viewpoint condition;

(iii) "navigation $\times$ viewpoint" interaction $[F(1,64)=$ 58.604; $P<.0001]$ : subjects performed better in passive than active navigation in the aerial-level viewpoint condition (i.e., a negative effect of the active condition); thus the reverse was observed in the ground-level viewpoint condition (i.e., the active condition had a positive effect in the ground-level viewpoint condition).

3.3. Errors in the Picture-Sorting Task. The ANOVA analysis revealed a significant effect for the following:

(i) navigation factor $[F(1,60)=3.900 ; P=.05]$ : subjects performed less well in the active than the passive viewpoint condition;

(ii) viewpoint factor $[F(1,60)=33.949 ; P<.0001]$ : subjects performed better in the ground-level than the aerial-level viewpoint condition;

(iii) "navigation $\times$ viewpoint interaction" was significant $[F(1,60)=8.807 ; P<.005]$ in the passive navigation 


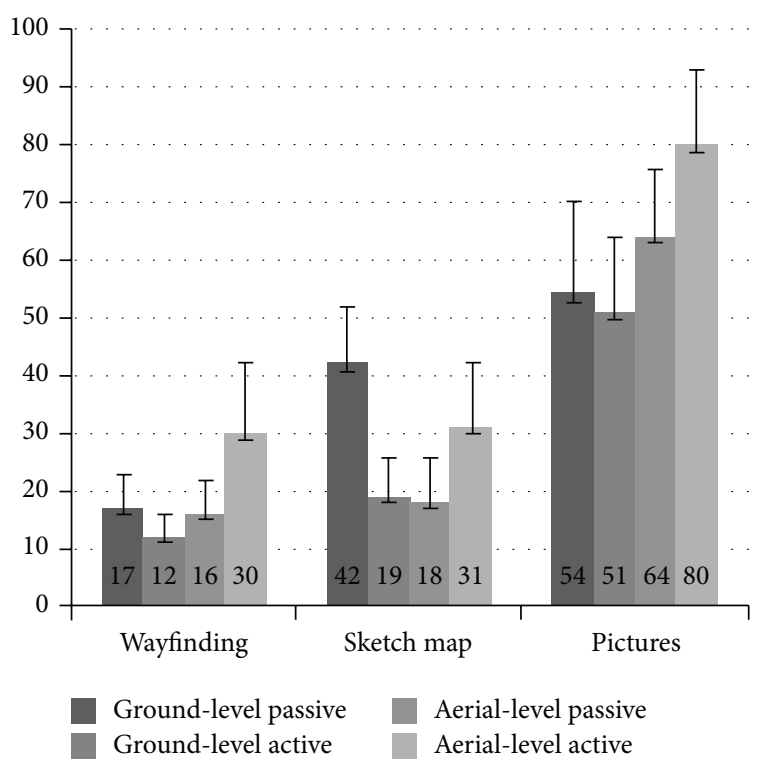

FIgURE 3: Means of errors (\%) for each recall task as a function of the Viewpoint and Navigation mode.

condition; subjects with a ground-level viewpoint performed as well as those with an aerial-level viewpoint. However, in the active navigation condition, subjects with a ground-level viewpoint performed better than those with an aerial-level viewpoint (i.e., the active condition had a negative effect in the aeriallevel viewpoint condition).

In summary, the ground-level viewpoint condition induced the best performance in the wayfinding and picturesorting tasks, whereas the aerial-level viewpoint condition induced the best performance in the sketch-mapping task. The active navigation mode had a negative impact on all tasks in the aerial-level viewpoint condition. In contrast, active navigation had a significant positive effect on both the wayfinding and sketch-mapping scores in the groundlevel viewpoint condition. Finally, active navigation did not significantly increase the picture-sorting score in the groundlevel condition, although it produced the highest scores for this task.

\section{Discussion}

The purpose of this study was to evaluate the effect of passive versus active navigation and ground-level versus aerial-level viewpoints on spatial knowledge in three different spatial recall tasks (wayfinding, sketch-mapping, and picturesorting). We expected that the best performances in wayfinding task would be obtained in active/ground-level navigation. Concerning the laboratory tasks, we hypothesized that the active/ground-level navigation would facilitate the performance in the picture-sorting task (egocentric frame-based) and passive/aerial-level navigation in the sketch-mapping task (allocentric frame-based).

Firstly, in terms of viewpoint manipulation, the findings were as expected in the various tasks. Indeed, our study demonstrated that the ground-level viewpoint condition induced the best performance in both wayfinding and picture-sorting tasks (egocentric frame-based tasks), whereas the aerial-level viewpoint condition induced the best performances in the sketch-mapping task (allocentric frame-based task). This may be due to the well-established principle of appropriate processing transfer [40], where learning performance is optimized when there is a perfect match between the learning and recall conditions. For wayfinding and picturesorting tasks, exploring the virtual district from the groundlevel viewpoint matched the retrieval conditions reasonably well (i.e., reference points are perceived during learning). The egocentric representations developed by subjects may therefore be used directly in the recall tasks. In the sketchmapping task, the frame of reference provided by the aeriallevel viewpoint (i.e., 4 meters above the ground) was closer to that required by the sketch-mapping task (a bird's eye view). It seems that participants in aerial-level mode build up survey knowledge of the previously followed path more easily. This can explain the better performance in the sketch-mapping task of subjects who had trained in the aerial-level viewpoint.

These observations are consistent with studies in a real environment, which demonstrated that different representations (i.e., egocentric versus allocentric) were involved depending on the learning source used and, more precisely, the learning viewpoint (e.g., direct navigation for an egocentric viewpoint and a map for an allocentric viewpoint) [6-9]. Our findings are also consistent with studies in VR systems, supporting the assertion that learning in groundlevel mode involves principally egocentric representations and facilitates an understanding of the route, while, in contrast, learning in aerial-level mode involves principally allocentric representations and promotes the acquisition of survey knowledge [36]. In general, this first demonstration confirmed that the viewpoint displacement metaphor is an important variable in VR systems, due to its mediating effects on the spatial representation and understanding of explored VE [35].

Secondly, regarding the issue of interaction between the navigation and viewpoint factors, our results confirmed our hypothesis that active navigation is mainly beneficial in the ground-level mode for the egocentric frame-based recall tasks. Indeed, the combination of active navigation mode and ground-level viewpoint enhanced performance in the wayfinding task and, to a lesser extent, in picture-sorting task. Active/ground-level learning markedly increased the subjects' performance in these two egocentric frame-based tasks compared to other combinations (i.e., active/aerial, passive/ground, or passive/aerial) and facilitated skill transfer from virtual to real environments in the wayfinding task. In contrast, combining the aerial-level viewpoint with the passive navigation mode led to better performance in the sketch-mapping task. As previously mentioned, the aeriallevel viewpoint facilitates the creation of allocentric representations, and our findings indicated that this effect was enhanced in the passive condition. This is probably why the aerial-level/active coupling appears to interfere with the development of an adequate mental representation for the execution of the sketch-mapping task. We concluded that, 
like the aerial-level mode, the passive navigation mode allows subjects to focus their attention on a global understanding of the environment, as previously asserted by Witmer et al. [36]. Another explanation about the interference of active navigation in the aerial perspective may be that the activity proposed in our experiment (i.e., with a joystick) does not provide complete idiothetic information (as would have been the case with a treadmill or a step-exercise device) which seems to play a key role in the survey strategy [33]. In future studies, it will be interesting to explore the relation between active navigation and cognitive processes involved in spatial learning. Indeed, spatial learning requires many cognitive processes that interact on the involvement of attention and working memory and active mental manipulation of spatial information [33].

Overall, these results have demonstrated that active navigation is mainly beneficial in association with a ground-level viewpoint (i.e., egocentric frame) in the learning phase for egocentric frame-based tasks. This confirmed the hypothesis that the benefits of active navigation are linked with egocentric frame-based situations and facilitates the establishment of accurate perception-action coupling. In this way, sensory-motor activity potentiates the correlation between visual (i.e., egocentric view of landmarks) and motor (i.e., direction changes) inputs, likely to be particularly beneficial for route knowledge acquisition (i.e., the second level in the L-R-S framework). This confirms the need to match viewpoints between learning (ground-level viewpoint) and recall (egocentric frame-based tasks) for active navigation to be beneficial.

Our study also confirms that the outcome of activepassive comparisons may depend on the type of spatial recall task and the measure employed (e.g., [13, 33]). These results provide an explanation for the discrepancies in the literature. Indeed, the beneficial effect of active navigation in VE studies has not been clearly established. As already detailed, some authors have reported a positive impact on spatial learning performances [18-20, 24-26] while others failed to find it [23, 27-30]. All of these studies used a groundlevel viewpoint during learning but different frame-based tasks (i.e., egocentric or allocentric). For example, Carassa et al. [32], who reported a positive effect of activity on route learning, used wayfinding task (i.e., an egocentric framebased task). In contrast, Gaunet et al. [28] and Wilson and Péruch [29], who found that active navigation did not have a positive effect, used sketch-mapping task (allocentric framebased task).

\section{Conclusion}

To conclude, our results provide relevant information in the passive/active debate. These findings offer a possible explanation for discrepancies in the literature, by confirming that active learning is particularly beneficial in egocentric framebased situations (learning with ground-level viewpoint and recall by wayfinding task) compared to allocentric framebased situations. However, further investigation is required to clarify the strategies and spatial knowledge brought into play by the navigation mode (active/passive) and the role of sensory-motor activity in potentiating the coupling between visual and motor information, and so promoting the transfer of spatial abilities. Particularly relevant applications may be envisaged in the field of cognitive assessment and neuropsychological rehabilitation for many medical conditions involving orientation and spatial memory difficulties [41]. In this context, it is highly relevant to identify the situations most likely to optimize the transfer of learning from virtual to real and to clarify the level of representation and spatial skills altered or preserved, depending on the type of pathology.

\section{Conflict of Interests}

The authors report no conflict of interests. The authors alone are responsible for the content and writing of the paper.

\section{References}

[1] A. W. Siegel and S. H. White, "The development of spatial representation of large scale environments," in Advances In Child Development and Behavior, H. Reese, Ed., vol. 10, pp. 1055, Academic Press, New York, NY, USA, 1975.

[2] J. Wiener, S. B. Büchner, and C. H. Hölscher, "Taxonomy of human wayfinding tasks: a knowledge-based approach," Spatial Cognition \& Computation, vol. 9, no. 2, pp. 1-14, 2009.

[3] D. R. Montello, "A new framework for understanding the acquisition of spatial knowledge in large-scale environments," in Spatial and Temporal Reasoning in Geographic Information Systems, M. J. Egenhofer and R. G. Golledge, Eds., pp. 143-154, Oxford University Press, New York, NY, USA, 1998.

[4] T. Ishikawa and D. R. Montello, "Spatial knowledge acquisition from direct experience in the environment: individual differences in the development of metric knowledge and the integration of separately learned places," Cognitive Psychology, vol. 52, no. 2, pp. 93-129, 2006.

[5] A. Berthoz, "Parietal and hippocampal contribution to topokinetic and topographic memory," Philosophical Transactions of the Royal Society B, vol. 352, no. 1360, pp. 1437-1448, 1997.

[6] P. Thorndyke and B. Hayes-Roth, "Differences in spatial knowledge acquired from maps and navigation," Cognitive Psychology, vol. 14 , pp. $560-589,1982$.

[7] D. H. Uttal, J. A. Fisher, and H. A. Taylor, "Words and maps: developmental changes in mental models of spatial information acquired from descriptions and depictions," Developmental Science, vol. 9, no. 2, pp. 221-235, 2006.

[8] L. S. Liben, "Environmental cognition through direct and representational experiences: a life-span perspective," in Environment, Cognition, and Action: An Integrated Approach, T. Garling and G. W. Evans, Eds., pp. 245-276, Oxford University Press, New York, NY, USA, 1991.

[9] D. H. Uttal, J. A. Fisher, and H. A. Taylor, "Words and maps: developmental changes in mental models of spatial information acquired from descriptions and depictions," Developmental Science, vol. 9, no. 2, pp. 221-235, 2006.

[10] T. Wolbers and M. Hegarty, "What determines our navigational abilities?" Trends in Cognitive Sciences, vol. 14, no. 3, pp. 138-146, 2010.

[11] P. P. Péruch, L. Belingard, and C. Thinus-Blanc, "Transfer of spatial knowledge from virtual to real environments," in Spatial 
Cognition II, LNAI, C. Freksa, W. Brauer, C. Habel, and K. F. Wender, Eds., vol. 1849, pp. 253-264, Springer, New York, NY, USA, 2000

[12] B. G. Witmer, J. H. Bailey, B. W. Knerr, and K. C. Parsons, "Virtual spaces and real world places: transfer of route knowledge," International Journal of Human Computer Studies, vol. 45, no. 4, pp. 413-428, 1996.

[13] P. Peruch and P. Wilson, "Active versus passive learning and testing in a complex outside built environment," Cognitive Processing, vol. 5, no. 4, pp. 218-227, 2004.

[14] N. Foreman, J. Stirk, J. Pohl et al., "Spatial information transfer from virtual to real versions of the Kiel locomotor maze," Behavioural Brain Research, vol. 112, no. 1-2, pp. 53-61, 2000.

[15] A. E. Richardson, D. R. Montello, and M. Hegarty, "Spatial knowledge acquisition from maps and from navigation in real and virtual environments," Memory and Cognition, vol. 27, no. 4, pp. 741-750, 1999.

[16] R. A. Ruddle, S. J. Payne, and D. M. Jones, "Navigating buildings in desk-top virtual environments: experimental investigations using extended navigational experience," Journal of Experimental Psychology, vol. 3, no. 2, pp. 143-159, 1997.

[17] D. Waller, E. Hunt, and D. Knapp, "The transfer of spatial knowledge in virtual environment training," Presence, vol. 7, no. 2, pp. 129-143, 1998.

[18] G. Wallet, H. Sauzéon, J. Rodrigues, and B. N'Kaoua, “Use of virtual reality for spatial knowledge transfer: Effects of passive/active exploration mode in simple and complex routes for three different recall tasks," in Proceedings of the ACM Symposium on Virtual Reality Software and Technology (VRST '08), pp. 175-178, October 2008.

[19] G. Wallet, H. Sauzéon, J. Rodrigues, F. Larrue, and B. N'Kaoua, "Virtual/real transfer of spatial learning: impact of activity according to the retention delay," Annual Review of CyberTherapy and Telemedicine, vol. 8, no. 1, pp. 115-118, 2010.

[20] G. Wallet, H. Sauzéon, P. A. Pala, F. Larrue, X. Zheng, and B. N'Kaoua, "Virtual/real transfer of spatial knowledge: benefit from visual fidelity provided in a virtual environment and impact of active navigation," Cyberpsychology, Behavior, and Social Networking, vol. 14, no. 7-8, pp. 417-423, 2011.

[21] J. J. Gibson, "Observations on active touch," Psychological Review, vol. 69, no. 6, pp. 477-491, 1962.

[22] S. L. Cohen and R. Cohen, "The role of activity in spatial cognition," in The Development of Spatial Cognition, R. Cohen, Ed., pp. 199-221, Lawrence Erlbaum Assoc, Hillsdale, NJ, USA, 1985.

[23] P. N. Wilson, N. Foreman, R. Gillett, and D. Stanton, "Active versus passive processing of spatial information in a computersimulated environment," Ecological Psychology, vol. 9, no. 3, pp. 207-222, 1997.

[24] P. P. Péruch, J. L. Vercher, and G. M. Gauthier, "Acquisition of spatial knowledge through visual exploration of simulated environments," Ecological Psychology, vol. 7, no. 1, pp. 1-20, 1995.

[25] K. H. James, G. K. Humphrey, T. Vilis, B. Corrie, R. Baddour, and M. A. Goodale, "Active and passive learning of threedimensional object structure within an immersive virtual reality environment," Behavior Research Methods, Instruments, and Computers, vol. 34, no. 3, pp. 383-390, 2002.

[26] J. Hahm, K. Lee, S.-L. Lim, S.-Y. Kim, H.-T. Kim, and J.-H. Lee, "Effects of active navigation on object recognition in virtual environments," Cyberpsychology and Behavior, vol. 10, no. 2, pp. 305-308, 2007.
[27] P. N. Wilson, "Active exploration of a virtual environment does not promote orientation or memory for objects," Environment and Behavior, vol. 31, no. 6, pp. 752-763, 1999.

[28] F. Gaunet, M. Vidal, A. Kemeny, and A. Berthoz, "Active, passive and snapshot exploration in a virtual environment: influence on scene memory, reorientation and path memory," Cognitive Brain Research, vol. 11, no. 3, pp. 409-420, 2001.

[29] P. N. Wilson and P. Péruch, "The influence of interactivity and attention on spatial learning in a desk-top virtual environment," Cahiers de Psychologie Cognitive, vol. 21, no. 6, pp. 601-633, 2002.

[30] N. Foreman, G. Sandamas, and D. Newson, "Distance underestimation in virtual space is sensitive to gender but not activity-passivity or mode of interaction," Cyberpsychology and Behavior, vol. 7, no. 4, pp. 451-457, 2004.

[31] B. M. Brooks, E. A. Attree, F. D. Rose, B. R. Clifford, and A. G. Leadbetter, "The specificity of memory enhancement during interaction with a virtual environment," Memory, vol. 7, no. 1, pp. 65-78, 1999.

[32] A. Carassa, G. Geminiani, F. Morganti, and D. Varotto, "Active and passive spatial learning in a complex virtual environment: the effects of efficient exploration," Cognitive Processing, vol. 3 , no. 4, pp. 65-81, 2002.

[33] E. R. Chrastil and W. H. Warren, "Active and passive contributions to spatial learning," Psychonomic Bulletin and Review, vol. 19, no. 1, pp. 1-23, 2012.

[34] N. Burgess, "Spatial cognition and the brain," Annals of the New York Academy of Sciences, vol. 1124, pp. 77-97, 2008.

[35] R. P. Darken and B. Peterson, "Spatial orientation, wayfinding, and representation," in Handbook of Virtual Environments: Design, Implementation, and Applications, K. M. Stanney, Ed., pp. 493-518, Lawrence Erlbaum Assoc, Hillsdale, NJ, USA, 2002.

[36] B. G. Witmer, W. J. Sadowski, and N. M. Finkelstein, "VE-based training strategies for acquiring survey knowledge," Presence, vol. 11, no. 1, pp. 1-18, 2002.

[37] J. P. Guilford and W. S. Zimmermann, Guilford-Zimmerman Aptitude Survey: Manual of Instructions and Interpretations, Consulting psychologists press, Minneapolis, Minn, USA, 1981.

[38] S. G. Vandenberg and A. R. Kuse, "Mental rotations, a group test of three-dimensional spatial visualization," Perceptual and Motor Skills, vol. 47, no. 2, pp. 599-604, 1978.

[39] F. D. Rose, B. M. Brooks, and A. A. Rizzo, "Virtual reality in brain damage rehabilitation: review," Cyberpsychology and Behavior, vol. 8, no. 3, pp. 241-262, 2005.

[40] C. D. Morris, J. D. Bransford, and J. J. Franks, "Levels of processing versus transfer appropriate processing," Journal of Verbal Learning and Verbal Behavior, vol. 16, no. 5, pp. 519-533, 1977.

[41] B. M. Brooks and F. D. Rose, "The use of virtual reality in memory rehabilitation: current findings and future directions," NeuroRehabilitation, vol. 18, no. 2, pp. 147-157, 2003. 

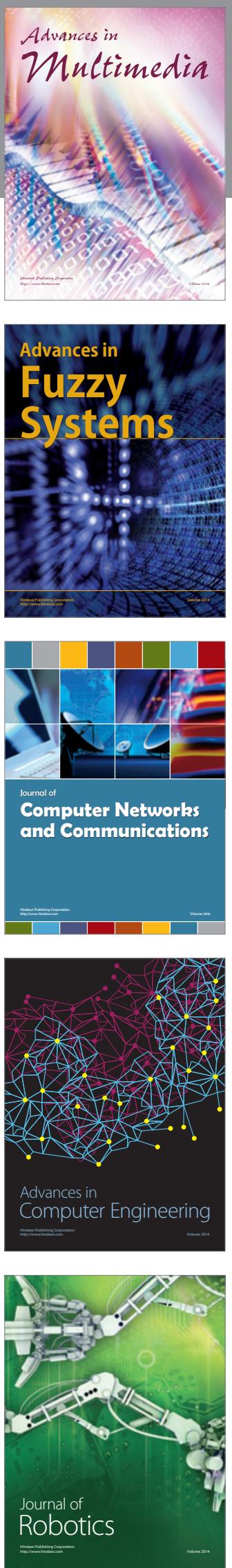

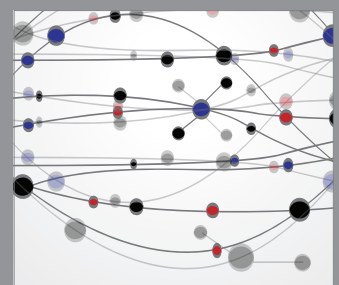

The Scientific World Journal
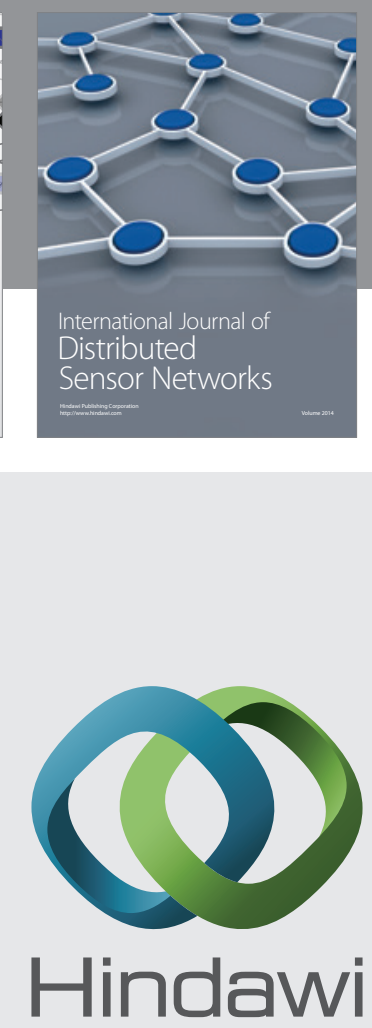

Submit your manuscripts at

http://www.hindawi.com
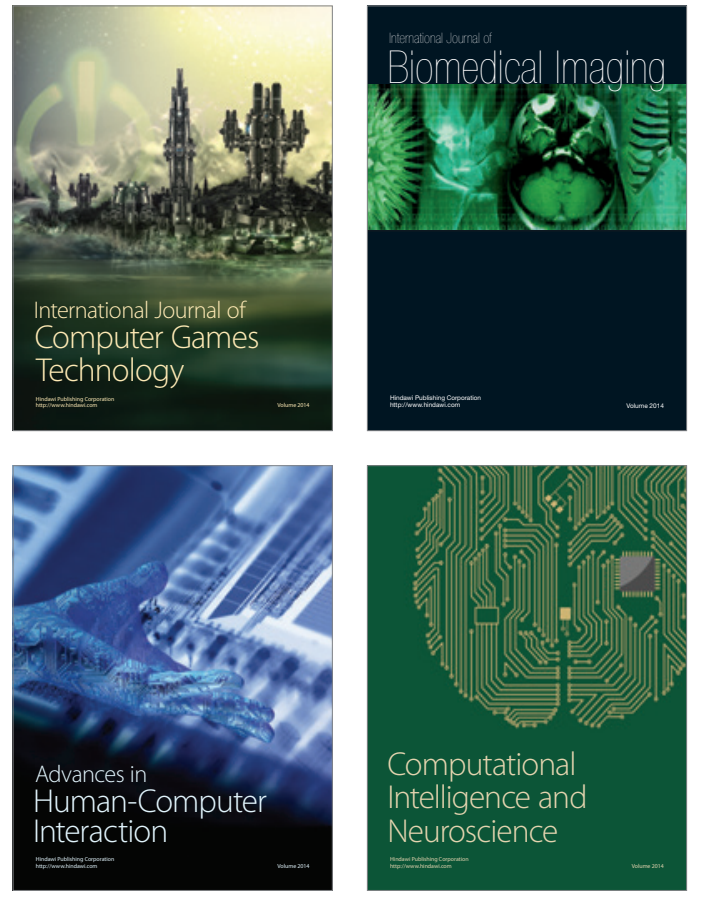
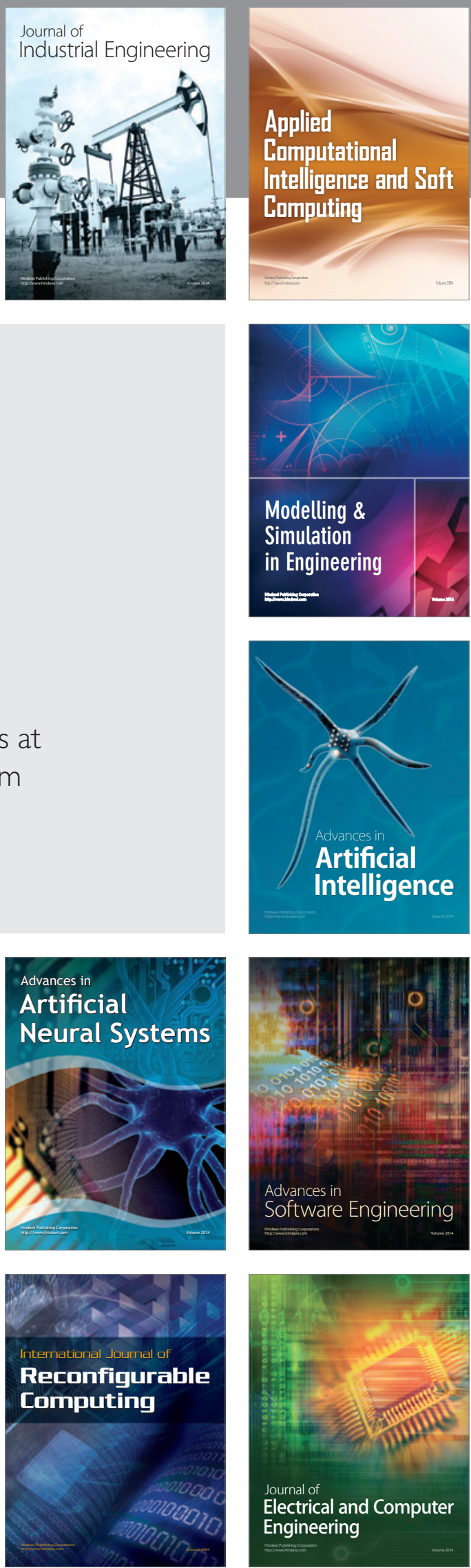